\title{
Propranolol and propranolol-LA in essential tremor: a double blind comparative study
}

\author{
LYNN CLEEVES, LESLIE J FINDLEY \\ From the MRC Neuro-Otology Unit, National Hospital, Queen Square, London and the Regional Centre for \\ Neurology and Neurosurgery, Oldchurch Hospital, Romford, Essex, UK
}

SUMMARY In a double blind, comparative study with 15 patients, a long-acting formulation of propranolol taken once daily (at doses of 160,240 and $320 \mathrm{mg}$ ), was shown to be as effective as conventional propranolol ( $80 \mathrm{mg}$ three times daily) in reducing the amplitude of essential tremor. The specific protocol employed demonstrated problems inherent in chronic pharmacological trials in essential tremor which have implications for future studies.

Propranolol is the drug of first choice in patients with essential tremor severe enough to require medication. The initial clinical observations of the effect of propranolol on essential tremor ${ }^{12}$ have been substantiated by a large number of controlled clinical trials. $^{3-6}$ These studies have shown that a significant mean reduction in tremor amplitude can be achieved on total daily doses of propranolol of between 120 and $240 \mathrm{mg}$. However, there is wide variability in individual responsiveness to propranolol even at high doses $^{7}$ and the clinical response is often incomplete. Approximately $70 \%$ of patients achieve symptomatic control and mean reductions in tremor amplitude of up to $60 \%$ have been reported. ${ }^{5-8}$ It is not possible to predict which patients will respond best, and there is no correlation between plasma levels of orally administered propranolol and tremorlytic effect. ${ }^{69}$

A long-acting formulation of propranolol (Inderal LA) (LA) is now available, allowing a once daily dosage and giving steady state plasma levels over 24 hours. ${ }^{10}$ Single daily doses of propranolol-LA and equivalent, but divided, doses of conventional propranolol (CP) produce similar steady state blood levels and a similar degree of beta-blockade throughout a 24 hour period. ${ }^{10-12}$ However, the marked variation in blood levels associated with repeated single dose therapy is avoided by the long-acting preparation. On theoretical grounds, LA should be as effective as the equivalent divided doses of $\mathrm{CP}$ in the management of essential tremor and may have the

Address for reprint requests: Dr L J Findley, MRC Neuro-Otology Unit, National Hospital, Queen Square, London WC1 3BG, UK.

Received 6 July 1987 and in revised form 21 September 1987. Accepted 22 September 1987 important clinical advantage of improved compliance and greater convenience to patients. ${ }^{13}$

In an initial study, Koller ${ }^{14}$ showed that LA substituted in equivalent doses for CP provided equivalent control of tremor in patients with essential tremor and was preferred by the majority.

In the present study, the efficacy of different single daily doses of LA were compared with placebo and a dose of CP ( $80 \mathrm{mg}$ three times daily) which has been previously shown to significantly attentuate essential tremor. $^{6}$ In addition, the time course of the control of tremor was assessed by testing patients at times of maximum (peak) and minimum (trough) drug effect.

\section{Patients}

Diagnosis of essential tremor was made on the basis of a history of postural and action tremor of the hands in the absence of other neurological signs and obvious aetiological factors (such as hyperthyroidism, alcoholism, betaadrenergic medication). Patients with a history of congestive cardiac failure, heart block and asthma were excluded.

Twenty three patients entered the study. Seventeen had not previously taken medication for tremor. Six who were on regular propranolol therapy were asked to withdraw the drug 2 weeks prior to starting the trial medication.

One patient was withdrawn from the study after an adverse reaction to treatment (see results) and another withdrew claiming a worsening of tremor on treatment. A further six patients withdrew for personal reasons. Clinical details of the 15 patients completing the trial are given in table 1 .

\section{Methods}

The trial was conducted according to a double blind, crossover design. There were five treatments consisting of conventional propranolol ( $80 \mathrm{mg}$ three times daily), propranolol-LA $(160,240$, and $320 \mathrm{mg}$ once daily) and 
Table 1 Patient details

\begin{tabular}{|c|c|c|c|c|c|}
\hline Patient & Sex & $\begin{array}{l}\text { Age } \\
\text { (yr) }\end{array}$ & $\begin{array}{l}\text { Duration } \\
\text { of tremor } \\
(y r)\end{array}$ & $\begin{array}{l}\text { Baseline } \\
\text { magnitude } \\
\left(g \times 10^{-3}\right)\end{array}$ & $\begin{array}{l}\text { Tremor } \\
\text { frequency } \\
(\mathrm{Hz})\end{array}$ \\
\hline 1 & $\mathbf{M}$ & 47 & 12 & $15 \cdot 5$ & $7 \cdot 1$ \\
\hline 2 & $\mathbf{M}$ & 18 & 10 & $22 \cdot 8$ & $5 \cdot 4$ \\
\hline 3 & $\mathbf{F}$ & 55 & 50 & 37.6 & 6.9 \\
\hline 4 & $\mathbf{M}$ & 21 & 20 & 46.0 & 6.5 \\
\hline 5 & $\mathbf{M}$ & 21 & 5 & $7 \cdot 9$ & 9.0 \\
\hline 6 & $\mathbf{F}$ & 46 & 40 & $12 \cdot 0$ & $7 \cdot 9$ \\
\hline 8 & $\mathbf{F}$ & 68 & 30 & $181 \cdot 3$ & $5 \cdot 4$ \\
\hline 9 & $\mathbf{M}$ & 27 & 12 & $18 \cdot 9$ & $7 \cdot 0$ \\
\hline 14 & $\mathbf{F}$ & 59 & 10 & $180 \cdot 4$ & $5 \cdot 5$ \\
\hline 16 & $\mathbf{F}$ & 62 & 10 & $402 \cdot 2$ & $6 \cdot 1$ \\
\hline 18 & $\mathbf{M}$ & 64 & 10 & 11.9 & $6 \cdot 1$ \\
\hline 19 & $\mathbf{M}$ & 73 & 5 & $37 \cdot 9$ & $5 \cdot 5$ \\
\hline 21 & $\mathbf{F}$ & 59 & 6 & $342 \cdot 8$ & $5 \cdot 9$ \\
\hline 22 & $\mathbf{M}$ & 66 & 10 & 83.4 & 5.9 \\
\hline 23 & $\mathbf{F}$ & 44 & 15 & 15.8 & $6 \cdot 7$ \\
\hline
\end{tabular}

placebo, given in randomised order for 3 weeks each. The regimen was identical for each treatment period and consisted of three capsules (taken in the morning only) and three tablets (one taken three times daily). During LA treatment, the total dose was contained in the three morning capsules and the tablets were placebos. During CP treatment each tablet contained $80 \mathrm{mg}$ of drug and the morning capsules were placebos.

Patients were assessed (see below) at the time of entry into the trial and at the end of each treatment period. On the day of testing, patients were asked to withhold their morning dose and were assessed in the laboratory before ("trough"), and after ("peak"), their medication. At the trough assessment, levels of both CP and LA would be expected to be minimum (approximately $12 \mathrm{~h}$ and $24 \mathrm{~h}$ respectively since last dose). Peak assessment was carried out $2 \cdot 5 \mathrm{~h}$ after the morning dose when both formulations of propranolol were expected to be exerting maximal effect.

Patients were asked to abstain from caffeine, tobacco and alcohol for at least $12 \mathrm{~h}$ prior to testing.

\section{Tremor assessment}

Before testing, patients were given at least 15 min rest in a quiet room to reduce the possibility of transient fluctuations in tremor amplitude resulting from stress, cold, etc encountered on the journey to hospital. Patients were asked to give details of any side effects experienced during the treatment period. Tremor was assessed by accelerometry, clinical rating, patient self rating, and manual performance.

Objective measures Miniature piezo-resistive linear accelerometers were attached, their sensitive axes oriented in the vertical plane, to the dorsal surface of each hand, in the second interspace, $1 \mathrm{~cm}$ proximal to the metacarpophalangeal joints. Tremor was recordec with the patient seated, forearms supported up to the wrists and hands outstretched horizontally in pronated posture. Three separate tremor recordings of $1 \mathrm{~min}$ duration were obtained at $5 \mathrm{~min}$ intervals. The hands were allowed to rest freely between recordings to minimise the effects of fatigue. Accelerometric signals were amplified and analysed on-line using a Hewlett Packard 5420-A signal analyser according to a method previously described. ${ }^{15}$ Tremor was characterised by frequency $(\mathrm{Hz})$ of the dominant peak and its amplitude scaled in rms acceleration, the unit of acceleration being taken as $1 \mathrm{~g}\left(\mathrm{~g}=981 \mathrm{~cm} / \mathrm{s}^{2}\right)$.

Clinical rating Postural tremor of the hands was graded clinically, by the same investigator, on a scale from zero (no observable tremor) to 5 (severe, self-injuring tremor). Scores from the two hands were summed (that is, maximum score 10).

Self-rating Patients were asked to give an overall assessment of the severity of their tremor during certain activities of daily living (writing, carrying cup and saucer, use of knife and fork) throughout the treatment period. The subjective scale was graded from zero (no tremor experienced) to 5 (maximum level of tremor experienced by the patient before treatment).

Performance tests Patients were asked to copy a short sentence and to trace inside an archimedes spiral. Test samples were identified by code only and were rated by three assessors who had no knowledge of the code. Scores ranged from zero (perfectly legible, no errors) to 5 (illegible, many errors) for both writing and tracing. For a given sample, the scores of all three assessors were added (that is, maximum 30 ).

\section{Heart rate, blood pressure and FEVI measurement}

On completion of tremor assessment, measurements of heart rate (beats $/ \mathrm{min}$ ) and systolic and diastolic blood pressure $(\mathrm{mm} \mathrm{Hg})$ were obtained after a $5 \mathrm{~min}$ period of rest in the supine position and again after $1 \mathrm{~min}$ of standing. Heart rate measured in the supine position was subtracted from heart rate after standing to give a single measure of standing tachycardia.

As an index of respiratory capacity, forced expiratory volume (litres) in one second (FEV1) was monitored using a standard vitalograph apparatus.

Finally, a $5 \mathrm{ml}$ sample of venous blood was taken for estimation of plasma propranolol levels.

After completing the assessment procedure, patients were asked to take their usual morning tablet and capsules. The procedure (with the exception of self rating) was then repeated $2.5 \mathrm{~h}$ later when plasma levels of CP and $\mathrm{LA}^{10}$ and tremorlytic effect of $\mathrm{CP}^{16}$ would be expected to be approaching maximum.

\section{Statistical analysis}

Overall effects of treatment on measures of tremor, heart rate, blood pressure and FEV1 were assessed by Friedman's 2-way analysis of variance. Comparisons of interest between pairs of means were made using the Wilcoxon test. Correlation between objective measures of tremor reduction and plasma propranolol levels was evaluated by Spearman's $r$ test.

Owing to computer malfunction, objective measures of tremor were not available for patients 3 and 4 on one occasion. Their data were therefore excluded from analysis of variance (that is, $n=13$ ). All other measures for these patients (self rating, clinical rating, manual performance) were unaffected and are included in the data analysis $(n=15)$.

\section{Results}

\section{Objective measures of tremor}

At trough, all drug treatments produced significantly $(p<0.02)$ lower tremor magnitudes than placebo (fig 



Fig 1 Mean (+/-SEM) tremor levels expressed in units of acceleration $(A)$ and as percentage of individual pre treatment levels $(B)$ during the different phases of the study. (T-Trough; P-Peak).

1a) and all except LA160 produced significantly $(p<0.01)$ greater percentage reductions in tremor (percent of pre treatment values) (fig $1 \mathrm{~b}$ ).

At peak, all active treatments (but not placebo) produced significantly $(p<0.05)$ lower tremor magnitudes than pre treatment values (fig 1). $\mathrm{CP}$ and all doses of LA produced lower mean tremor levels than placebo. However, this reached statistical significance ( $p<0.05)$ only for LA240 (absolute reduction) and LA320 (percentage reduction).
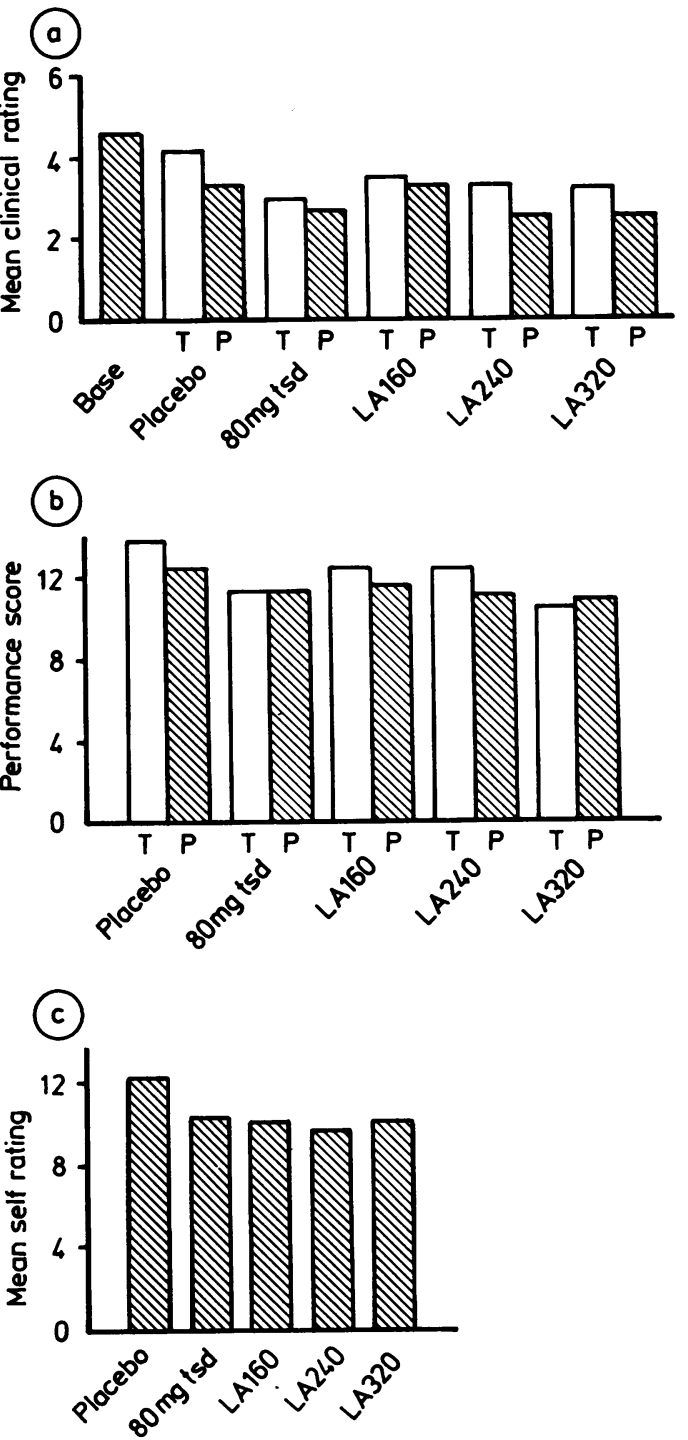

Fig 2 Mean clinical rating $(A)$, performance scores $(B)$ and self-rating $(C)$ for the different phases of the study.

There were no significant differences between trough and peak measures for any of the active treatments. Placebo, however, produced significantly lower $(p<0.01)$ levels of tremor at peak assessment compared with trough.

Clinical rating Analysis of variance indicated a significant difference $(p<0.01)$ between treatments at both trough and peak assessments (fig 2). At trough, all active treatments except LA160 produced significantly lower scores than placebo $(p<0.05)$. At 
peak, there was no significant difference between scores on CP and LA but only LA240 was significantly better than placebo $(\mathrm{p}<0.05)$.

Differences between scores at trough and peak were significant $(\mathrm{p}<0.05)$ for placebo, LA240 and LA320. Patients' self-rating Scores for all active treatments were significantly lower than for placebo $(p<0.05)$ with no differences between CP and LA (fig 2).

Performance tests At trough assessment, CP and LA320 produced significantly lower performance scores than placebo $(p<0.01)$ (fig 2$)$. There were no differences between placebo and any of the active treatments at peak assessment. Differences between scores at trough and peak were significant $(p<0.05)$ for placebo only.

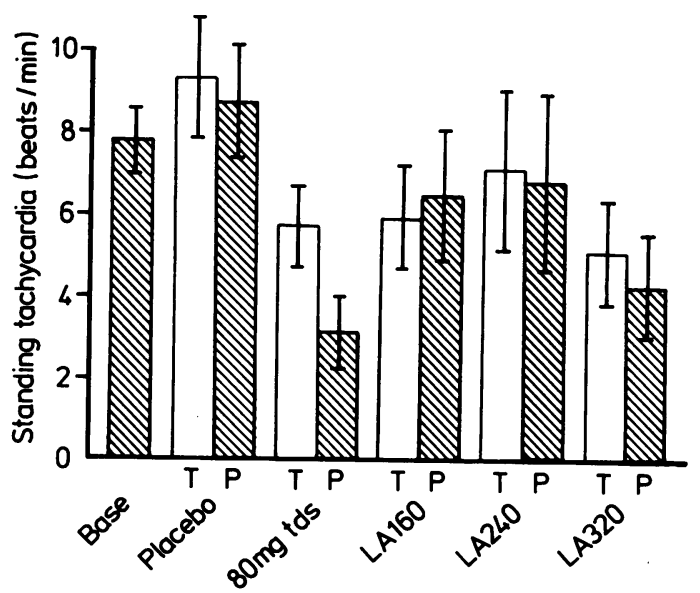

Fig 3 Mean increases in heart rate on standing (beat/min) $+/-S E M$ during the different phases of the study.

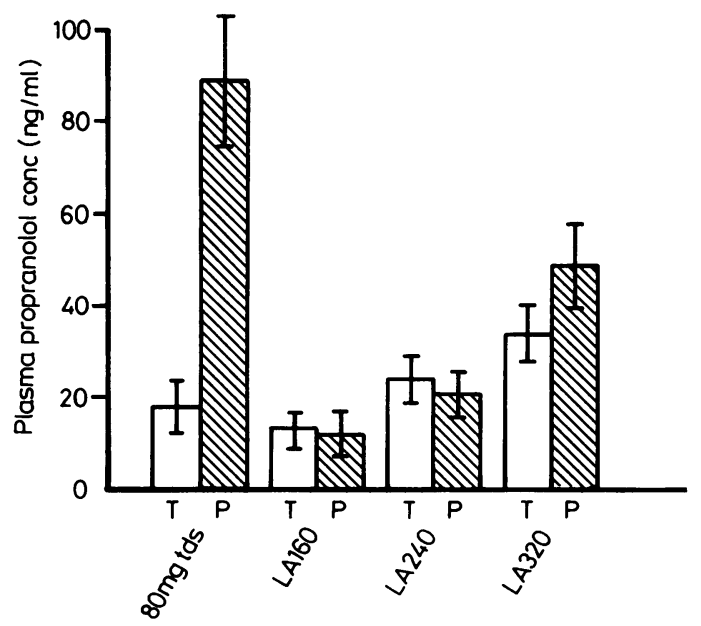

Fig 4 Mean plasma levels of propranolol $(\mathrm{ng} / \mathrm{ml})$ and SEM at trough and peak assessments.
Heart rate, blood pressure, FEVI At trough, all active treatments except LA240 significantly reduced standing tachycardia $(p<0.05)$ (fig 3$)$. At peak, only CP and LA320 were significantly different from placebo $(p<0.05)$. Standing tachycardia at peak assessment was significantly lower than at trough assessment for CP. There were no differences between peak and trough measures for placebo or any dose of LA.

Mean systolic and diastolic blood pressure measures did not differ between treatments. There were no differences between lying and standing blood pressure measures nor between peak and trough measures at any phase of the study. Similarly, FEV1 was unaffected by any treatment at trough or peak.

Plasma levels Mean plasma levels of propranolol at trough and peak assessments are shown in fig 4. At trough, mean drug level on CP was not significantly different from drug levels on LA160 and LA240 but was significantly lower than LA320 $(p<0.02)$. At peak, CP produced significantly higher plasma levels than all doses of LA $(P<0.01)$. LA320 produced significantly higher levels than LA160 and LA240 $(\mathrm{p}<0.02)$. There were no differences between trough and peak levels on LA160 and LA240. There was a small, significant difference between trough and peak levels on LA320 $(p<0.02)$ and a marked difference $(\mathrm{p}<0.01)$ on CP.

Side effects One patient was withdrawn from the trial after developing a severe skin eruption during the first treatment period (LA320). This resolved one week after discontinuing treatment. One patient claimed that her tremor was made worse by the first treatment (LA320) but was unwilling to attend for objective evaluation of this.

A number of mild side effects were reported by patients completing the trial. These are presented in table 2.

\section{Discussion}

The results confirm those of Koller $^{14}$ who found LA to be as effective as $\mathrm{CP}$ in reducing levels of essential tremor from pretreatment values. In the present,

Table 2 Side effects in patients completing the trial

\begin{tabular}{lll}
\hline Treatment & Reported side effects & $\begin{array}{l}\text { Incidence } \\
\text { (no.of patients) }\end{array}$ \\
\hline Placebo & $\begin{array}{l}\text { Breathlessness, dizziness, } \\
\text { palpitations }\end{array}$ & 3 \\
80 mg tds & $\begin{array}{l}\text { Headache, nausea, dizziness } \\
\text { LA160 }\end{array}$ & $\begin{array}{l}\text { Breathlessnes, tiredness, } \\
\text { diarrhoea, tingling sensation } \\
\text { in arms }\end{array}$ \\
LA240 & $\begin{array}{c}\text { Headache, dizziness, tiredness, } \\
\text { diarrhoea }\end{array}$ & 4 \\
LA320 & $\begin{array}{c}\text { Breathlessness, headache, dizziness, } \\
\text { tiredness, depression, hot flushes }\end{array}$ & 5 \\
\hline
\end{tabular}


placebo controlled, study all active treatments (at peak assessment) produced lower levels of tremor than placebo, but this reached statistical significance only for LA240 (absolute units) and LA320 (percentage reduction).

A number of previous clinical trials of $\mathrm{CP}$ at the dosage used in the present study have found clear and statistically significant reductions in tremor when compared with placebo. ${ }^{346}$ Though trials showing no difference between CP (at doses less than $240 \mathrm{mg}$ daily) and placebo have been reported, ${ }^{1718}$ inspection of the present data suggests that the absence of a statistically significant tremorlytic effect, against placebo, with CP does not represent a failure of response to the drug.

A placebo effect is invariably found in clinical trials in essential tremor. Where the placebo effect has been quantified, this has ranged from $4.9 \%$ on chronic administration ${ }^{4}$ to $12 \%$ after single oral dose. ${ }^{5}$ In the present study, tremor levels recorded at trough assessment showed no difference between placebo and baseline (that is, no placebo effect) whereas all active treatments produced significantly lower levels of tremor than placebo. This provides evidence for tremorlytic efficacy of both CP and LA even at 12 and 24 hours respectively since last dose: However, when patients were asked to take their morning medication in the laboratory setting and re-tested at the time of peak effect, a large and statistically significant placebo effect (mean reduction $32.4 \%$ ) was observed, thus diminishing the apparent effect of active treatments which themselves showed no difference between trough and peak tremor levels.

These findings on objective measures are substantiated by clinical rating and performance data. At trough, all active treatments except LA160 produced significantly lower clinical scores than placebo. At peak, however, only LA240 produced a significant effect. Similarly, whilst CP and LA320 produced performance scores significantly lower than placebo at trough, no drug was better than placebo at peak assessment.

The results from trough assessment demonstrate that both $C P$ and LA are effective in reducing the amplitude of essential tremor. However, the results at peak assessment are confounded by the large placebo effect which appears to be specific to this design in which acute drug effects are assessed on a background of chronic drug effects. In the placebo condition, the chronic placebo response (assessed at "trough") has diminished whilst an acute placebo response is clearly observed at "peak" (fig 1A). In all active conditions, a clear response is still seen at trough assessment and thus, little further change is observed at peak.

Of considerable importance is the finding that, according to the patients' own judgement (arguably the most relevant index of drug efficacy), all active treatments were clearly discriminable from, and judged equally superior to, placebo.

These findings serve to emphasise the problems inherent in the conduct of clinical trials in essential tremor and the importance of utilising a variety of measures for assessing drug efficacy. ${ }^{19}$ Essential tremor is a fluctuating phenomenon with a variable response to treatment. We have recently shown that day to day variability in amplitude of untreated essential tremor can be as much as 10 -fold in some patients. ${ }^{20}$ Furthermore, we found a tendency for a systematic reduction in tremor levels over repeated assessments. In view of this an initial, pre-treatment, measure which is usually taken as the "baseline" value, is likely to represent the highest levels of tremor experienced by the patient, rather than an "average" or representative level. Thus, the appropriate control for evaluation of drug effects in essential tremor would appear to be a randomised placebo condition. However, as the present study demonstrates, the placebo response itself can itself be affected by the mode of assessment.

As in previous studies, ${ }^{69}$ degree of tremor reduction was not correlated with plasma propranolol levels or with changes in standing tachycardia. Insofar as the latter measure is an index of beta-blockade, it can be said that the tremorlytic action and cardiac betablocking action of propranolol are independent. Indeed, in some patients maximum tremorlytic effect may occur at doses higher than those necessary for complete beta-blockade.

Tablet and capsule counts at the end of treatment periods revealed significantly better compliance for LA than for CP. This may account for the smaller tremor reduction seen in this study with propranolol $80 \mathrm{mg}$ three times daily compared the same dose of LA taken once daily.

In conclusion, propranolol-LA appears to have tremorlytic efficacy comparable with, and at the highest doses used ( 240 and $320 \mathrm{mg}$ ), superior to conventional propranolol. Better compliance with the once daily preparation offers an advantage over the standard preparation in the management of essential tremor.

\section{References}

1 Winkler GF, Young RR. The control of essential tremor by propranolol. Trans Am Neurol Assoc 1971;96: 66-8.

2 Sevitt I. The effect of adrenergic beta-receptor blocking drugs on tremor. Practitioner 1971;207:677-8.

3 Jefferson D, Jenner P, Marsden CD. Betaadrenoreceptor antagonists in essential tremor. $J$ Neurol Neurosurg Psychiatry 1979;42:904-9.

4 Larsen TA, Teravainen H. Beta-blockers in essential 
tremor. Lancet 1981;ii:533.

5 Calzetti S, Findley LJ, Gresty MA, Perucca E, Richens A. Effect of a single oral dose of propranolol on essential tremor: a double blind controlled study. Ann Neurol 1983;13:165-71.

6 Calzetti S, Findley LJ, Perucca E, Richens A. The response of essential tremor to propranolol: evaluation of clinical variables governing its efficacy on prolonged administration. J Neurol Neurosurg Psychiatry 1983;46:393-8.

7 Koller W. Dose-response relationship of propranolol in the treatment of essential tremor. Arch Neurol 1986; 43:42-3.

8 Winkler GF, Young RR. Efficacy of chronic propranolol therapy in action tremors of the familial, senile or essential varieties. $N$ Engl $J$ Med 1974;290: 984-8.

9 Jefferson D, Jenner P, Marsden SD. Relationship between plasma propranolol concentration and relief of essential tremor. J Neurol Neurosurg Psychiatry 1979;42:831-7.

10 Baber NS, McAinsh J, Young J, Smith R. Pharmacokinetic and pharmacodynamic studies with long-acting propranolol. Br J Clin Pharmacol 1978; 5:355P-356P.

11 Lamb R, Kennedy JG. Double blind cross over trial comparing "Inderal-LA" $160 \mathrm{mg}$ once daily and conventional "Inderal" $40 \mathrm{mg}$ four times daily in the treatment of angina pectoris. Br J Clin Pract 1982; suppl 20:65-8.

12 Zacharias FJ. "Inderal LA" once daily versus conventional "Inderal" twice daily in mild to moderate essential hypertension; three months biochemistry and blood levels of propranolol. $\mathrm{Br} J$ Clin Pract 1982;suppl 20:47-9.

13 Pearson RM. How to decide which beta-blocker should be chosen. Ploys to make patients take their tablets. MIMS Magazine 1983 (Feb 1st):20-5.

14 Koller W. Long-acting propranolol in essential tremor. Neurology 1985;35:108-10.

15 Cleeves L, Findley LJ. Beta-adrenoreceptor mechanisms in essential tremor: a comparative single dose study of the effect of a non-selective and a beta- 2 selective adrenoreceptor antagonist. $J$ Neurol Neurosurg Psychiatry 1984;47:976-82.

16 Koller W. Time course of a single oral dose of propranolol in essential tremor. Neurology 1985;35:1494-8.

17 Balla JI. Treatment of essential tremor with propranolol. Lancet 1973;i:205.

18 Sweet RD, Blumberg J, Lee JE, McDowell FH. Propranolol treatment of essential tremor. Neurology 1974;24:64-7.

19 Findley LJ, Cleeves L. Phenobarbitone in essential tremor. Neurology 1985;35:1784-7.

20 Cleeves L, Findley LJ. Variability in amplitude of untreated essential tremor. $J$ Neurol Neurosurg Psychiatry 1987;50:704-8. 